\title{
Inaugural editorial of CCF transactions on pervasive computing and interaction
}

\author{
Zhiwen $\mathrm{Yu}^{1} \cdot$ Anind Dey ${ }^{2}$ \\ Published online: 13 May 2019 \\ (c) China Computer Federation (CCF) 2019
}

Welcome to the CCF Transactions on Pervasive Computing and Interaction (CCF TPCI). We are very excited to launch the inaugural issue of our new publication. It is our great honor to serve the CCF TPCI.

The field of pervasive computing and interaction is evolving at an ever faster rate, with a promise to bring in revolutionary paradigms for computing models as well as to change the way we interact with machines. Rapid advancements in a variety of technologies such as crowd sensing, mobile sensing, sensory data mining, human-machine collaboration, brain-machine interaction and intelligent user interface have significantly contributed to the evolution of pervasive computing and interaction. Although there are several existing periodical related to CCF TPCI, e.g., ACM Transactions on Computer-Human Interaction, IEEE Transactions on Human-Machine Systems, and Elsevier Pervasive and Mobile Computing, CCF TPCI is the first journal that combines human-computer interaction and ubiquitous computing. Besides the traditional topics of these two fields, this journal especially focuses on the studies that try to fuse human, society, machine, and environment and provide intelligent interaction and services. Some of the key topics include cyber-physical-social systems, crowd sensing and computation, human-machine collaboration, etc.

CCF TPCI is dedicated to publishing rigorously peerreviewed, high-quality scientific articles covering all aspects of human-computer interaction and pervasive/ubiquitous computing. Computing technology is becoming increasingly pervasive which involves human, machine, and environment and it enables more advanced interactions. To foster the developments in the area, CCF TPCI provides a publication

Zhiwen Yu

zhiwenyu@nwpu.edu.cn

Anind Dey

anind@uw.edu

1 Northwestern Polytechnical University, Xi' an 710065, China

2 University of Washington, Seattle, WA 98105, USA and communication platform and welcomes contributions from researchers, professionals, and industrial practitioners. This journal covers a broad range of topics of interest, such as intelligent sensing, user interfaces, interaction models, mobile systems, wearable technologies, human-machine systems, and cyber-physical-social systems. We welcome original contributions, survey papers on new models, methodologies and tools in the covered topics. This journal is published on behalf of the China Computer Federation (CCF), and it is powered by the CCF technical committees on Pervasive Computing and Human-Computer Interaction. Each paper submitted is subjected to a thorough review procedure, and the publication decision made by the editorin-chief is fairly based on the reviewing comments and the associate editors' recommendations.

We will do our best to make sure CCF TPCI stays true to its aims and values. This premier issue includes articles that deal with topics ranging from activity recognition and human behavior understanding to augmented reality and human-computer interaction. The remaining three issues in 2019 will include articles dealing with topics such as crowd sensing, recommender system, human-AI collaboration, and innovative interaction approaches. We look forward to receiving research reports on operationally relevant topics.

Finally, we would like to take this opportunity to thank all the authors, reviewers, and Editorial Board members for their contributions. Particularly, we wish to thank the CCF TPCI Advisory Board for their support. Ultimately, the success of CCF TPCI depends on the submissions, suggestions and volunteer services we received from all of you. Thank you all, in advance. 


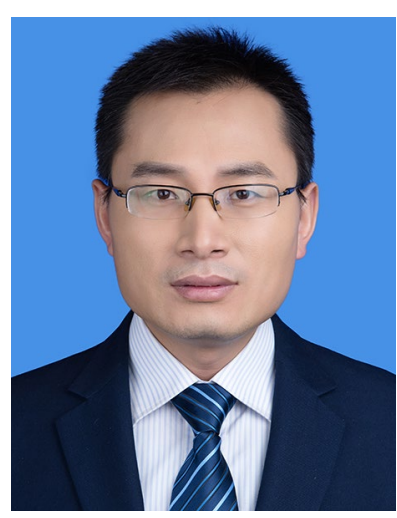

Zhiwen $\mathrm{Yu}$ is a professor of School of Computer Science and director of Institute of Ubiquitous and Intelligent Computing in Northwestern Polytechnical University, China. His research interests include ubiquitous computing, human-computer interaction, and mobile computing.

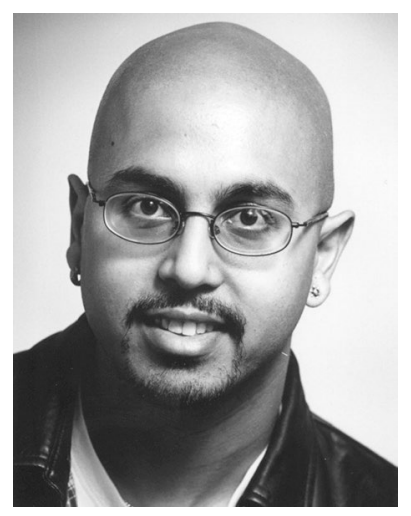

Anind Dey is a professor and dean of the Information School at the University of Washington, USA. His research interests include ubiquitous computing, contextaware computing, and humancomputer interaction. 